\title{
Meta-genome-wide association studies identify a locus on chromosome 1 and multiple variants in the MHC region for serum C-peptide in type 1 diabetes
}

\author{
Delnaz Roshandel ${ }^{1}$ - Rose Gubitosi-Klug ${ }^{2}$ - Shelley B. Bull ${ }^{3,4}$ • Angelo J. Canty ${ }^{5}$. Marcus G. Pezzolesi ${ }^{6}$. \\ George L. King ${ }^{7,8} \cdot$ Hillary A. Keenan ${ }^{7,8}$ - Janet K. Snell-Bergeon ${ }^{9}$. David M. Maahs ${ }^{9,10} \cdot$ Ronald Klein $^{11}$. \\ Barbara E. K. Klein ${ }^{11}$ - Trevor J. Orchard ${ }^{12} \cdot$ Tina Costacou $^{12}$ - Michael N. Weedon ${ }^{13}$ • DCCT/EDIC Research Group • \\ Richard A. Oram ${ }^{13,14}$. Andrew D. Paterson ${ }^{1,4}$
}

Received: 12 October 2017 / Accepted: 15 December 2017 / Published online: 5 February 2018

(C) The Author(s) 2018. This article is an open access publication

\begin{abstract}
Aims/hypothesis The aim of this study was to identify genetic variants associated with beta cell function in type 1 diabetes, as measured by serum C-peptide levels, through meta-genome-wide association studies (meta-GWAS).

Methods We performed a meta-GWAS to combine the results from five studies in type 1 diabetes with cross-sectionally measured stimulated, fasting or random C-peptide levels, including 3479 European participants. The $p$ values across studies were combined, taking into account sample size and direction of effect. We also performed separate meta-GWAS for stimulated ( $n=1303)$, fasting $(n=2019)$ and random $(n=1497)$ C-peptide levels.

Results In the meta-GWAS for stimulated/fasting/random C-peptide levels, a SNP on chromosome 1, rs559047 (Chr1:238753916, T>A, minor allele frequency [MAF] 0.24-0.26), was associated with C-peptide $\left(p=4.13 \times 10^{-8}\right)$, meeting the genome-wide significance threshold $\left(p<5 \times 10^{-8}\right)$. In the same meta-GWAS, a locus in the MHC region (rs9260151) was
\end{abstract}

A complete list of participants in the DCCT/EDIC Research Group is presented in the Supplementary Material published online for the article in N Engl J Med 2015;372:1722-1733.

Electronic supplementary material The online version of this article (https://doi.org/10.1007/s00125-018-4555-9) contains peer-reviewed but unedited supplementary material, which is available to authorised users.

Andrew D. Paterson

andrew.paterson@sickkids.ca

1 Genetics and Genome Biology Program, Peter Gilgan Centre for Research and Learning (PGCRL), The Hospital for Sick Children, 686 Bay Street, Toronto, ON M5G 1H3, Canada

2 University Hospitals Case Western Medical Center, Cleveland, $\mathrm{OH}$, USA

3 Lunenfeld-Tanenbaum Research Institute, Sinai Health System, Toronto, ON, Canada

4 Dalla Lana School of Public Health, University of Toronto, Toronto, ON, Canada

5 Department of Mathematics and Statistics, McMaster University, Hamilton, ON, Canada

6 Division of Nephrology and Hypertension, Diabetes and Metabolism Center, University of Utah, Salt Lake City, UT, USA
7 Research Division, Joslin Diabetes Center, Boston, MA, USA

8 Department of Medicine, Harvard Medical School, Boston, MA, USA

9 Barbara Davis Center for Diabetes, University of Colorado Anschutz Medical Campus, Aurora, CO, USA

10 Department of Paediatrics, Stanford School of Medicine, Stanford, CA, USA

11 Department of Ophthalmology and Visual Sciences, University of Wisconsin, Madison, WI, USA

12 Department of Epidemiology, Graduate School of Public Health, University of Pittsburgh, Pittsburgh, PA, USA

13 Institute for Biomedical and Clinical Science, University of Exeter Medical School, Exeter, UK

14 National Institute for Health Research, Exeter Clinical Research Facility, Exeter, UK 


\section{Research in context}

\section{What is already known about this subject?}

- Little is known about the genetics of beta cell function in type 1 diabetes

\section{What is the key question?}

- What are the genetic variants that affect beta cell function in type 1 diabetes, as measured using C-peptide levels?

\section{What are the new findings?}

- A single locus on chromosome 1 and multiple variants in the $\mathrm{MHC}$ region are associated with beta cell function in type 1 diabetes

- Some genetic variants associated with the severity of beta cell loss are distinct from those associated with the development of type 1 diabetes

\section{How might this impact on clinical practice in the foreseeable future?}

- These findings could eventually provide insight into mechanisms of beta cell loss and opportunities to preserve beta cell function in type 1 diabetes

close to the genome-wide significance threshold (Chr6:29911030, C>T, MAF 0.07-0.10, $p=8.43 \times 10^{-8}$ ). In the stimulated Cpeptide meta-GWAS, rs61211515 (Chr6:30100975, T/-, MAF 0.17-0.19) in the MHC region was associated with stimulated Cpeptide $\left(\beta[\mathrm{SE}]=-0.39[0.07], p=9.72 \times 10^{-8}\right)$. rs61211515 was also associated with the rate of stimulated C-peptide decline over time in a subset of individuals $(n=258)$ with annual repeated measures for up to 6 years $(p=0.02)$. In the meta-GWAS of random C-peptide, another MHC region, SNP rs3135002 (Chr6:32668439, C>A, MAF 0.02-0.06), was associated with Cpeptide $\left(p=3.49 \times 10^{-8}\right)$. Conditional analyses suggested that the three identified variants in the MHC region were independent of each other. rs9260151 and rs3135002 have been associated with type 1 diabetes, whereas rs559047 and rs61211515 have not been associated with a risk of developing type 1 diabetes.

Conclusions/interpretation We identified a locus on chromosome 1 and multiple variants in the MHC region, at least some of which were distinct from type 1 diabetes risk loci, that were associated with C-peptide, suggesting partly non-overlapping mechanisms for the development and progression of type 1 diabetes. These associations need to be validated in independent populations. Further investigations could provide insights into mechanisms of beta cell loss and opportunities to preserve beta cell function.

Keywords C-peptide $\cdot$ Genome-wide association study $\cdot$ Insulin-secreting cells $\cdot$ Single nucleotide polymorphism $\cdot$ Type 1 diabetes

$\begin{array}{ll}\text { Abbreviations } \\ \text { CACTI } & \text { Coronary Artery Calcification in Type 1 Diabetes } \\ \text { Chr } & \text { Chromosome } \\ \text { EDC } & \text { Pittsburgh Epidemiology of Diabetes Complications } \\ \text { eQTL } & \text { Expression quantitative trait loci } \\ \text { FDR } & \text { False discovery rate } \\ \text { GRS } & \text { Genetic risk score } \\ \text { GWAS } & \text { Genome-wide association study } \\ \text { LD } & \text { Linkage disequilibrium } \\ \text { MAF } & \text { Minor allele frequency } \\ \text { SFDR } & \text { Stratified false discovery rate } \\ \text { T1DGC } & \text { Type 1 Diabetes Genetics Consortium } \\ \text { WESDR } & \text { Wisconsin Epidemiologic Study of Diabetic } \\ & \text { Retinopathy }\end{array}$

\section{Introduction}

Type 1 diabetes develops when pancreatic beta cells fail to produce sufficient insulin to maintain euglycaemia [1]. Cpeptide measurement after a standard meal (stimulated Cpeptide) is the primary way to evaluate insulin secretion in type 1 diabetes [2, 3]. However, fasting/random C-peptide measurements are less demanding and feasible to obtain in large population samples. The alpha and beta chains of the proinsulin molecule are joined by C-peptide, which is removed during insulin secretion. Therefore, C-peptide is co-secreted with insulin in an equimolar ratio. Unlike insulin, C-peptide undergoes little first-pass clearance by the liver, has a much longer half-life and is not interfered with by exogenous insulin $[2,3]$. 
Type 1 diabetes is a heterogeneous disease that can occur at any age. C-peptide levels vary at diagnosis. Early-onset type 1 diabetes (age $<2-5$ years) is associated with rapid beta cell loss, the presence of high-risk MHC type 1 diabetes genetic susceptibility and high titres of autoantibodies compared with late-onset disease [4]. A large proportion of people with type 1 diabetes have detectable $\mathrm{C}$-peptide years after diagnosis, indicating that some beta cells are still functional [2, 5-8]. Sensitive assays have detected stimulated C-peptide in 73$100 \%$ of people with type 1 diabetes for $\geq 30$ years $[7,8]$.

C-peptide preservation has been reported to be associated with favourable metabolic and clinical outcomes in type 1 diabetes. Individuals with preserved C-peptide levels maintain better long-term glycaemic control despite a lower exogenous insulin dose per body weight [3]. They are also at lower risk for both hypoglycaemia and diabetic complications, which are major health problems [3, 9-12]. The association of C-peptide with long-term diabetic complications (i.e. retinopathy and nephropathy) remains significant after adjusting for long-term glycaemic control, suggesting that at least part of the effect of C-peptide on diabetic complications is independent of its effect on glycaemic control (or, alternatively, that frequency of blood glucose $/ \mathrm{HbA}_{1 \mathrm{c}}$ measurements is suboptimal) [3, 9]. However, association of C-peptide levels with long-term diabetic complications has not been observed in other studies [13].

There have been no family/twin studies of C-peptide levels in type 1 diabetes. In the Diabetes Control and Complications Trial (DCCT), investigations of detectable stimulated Cpeptide levels in participants with type 1 diabetes $(n=98)$ and their first-degree affected relatives $(n=109)$ could not reach conclusions because of an insufficient sample size (see electronic supplementary material [ESM] Tables 1, 2). To date, more than 50 type 1 diabetes risk loci have been identified. The majority are thought to be involved in the immune system, and many are shared with other autoimmune diseases [14]. However, there have been no genome-wide association studies (GWAS) of C-peptide in type 1 diabetes (www.ebi.ac. uk/gwas, accessed 1 July, 2017) and candidate geneassociation studies have investigated a limited number of genes in small populations with no replication in independent studies [15]. It is clear from other diseases that factors for the risk of a disease and its progression can be independent [16]. Here, we aimed to identify genetic loci associated with residual beta cell function in type 1 diabetes as measured by $\mathrm{C}$ peptide through meta-analysis of GWAS.

\section{Methods}

\section{Study design}

We performed meta-GWAS of stimulated, fasting and random (non-fasting) C-peptide separately in European (confirmed by population structure analysis) participants from the following five type 1 diabetes studies (Fig. 1):

- Stimulated C-peptide: the DCCT, including: (1) primary cohort; (2) secondary cohort with diabetes duration 15 years; and (3) secondary cohort with diabetes duration 5-15 years

- Fasting C-peptide: (1) the DCCT (all); (2) the Coronary Artery Calcification in Type 1 Diabetes (CACTI) study; and (3) the Pittsburgh Epidemiology of Diabetes Complications (EDC) study

- Random C-peptide: (1) the Joslin 50-Year Medalist study; and (2) the Wisconsin Epidemiologic Study of Diabetic Retinopathy (WESDR)

In the analysis of stimulated C-peptide, DCCT participants were divided into three groups (a primary cohort and a secondary cohort with diabetes duration of 1-5 and 5-15 years), since the range of stimulated $\mathrm{C}$-peptide levels differed among the three groups based on the inclusion/exclusion criteria. In contrast, because all DCCT participants had fasting C-peptide levels of $\leq 0.2 \mathrm{pmol} / \mathrm{ml}$, the fasting C-peptide analysis was performed with all participants as a single group ('all').

Another meta-analysis was performed to combine the GWAS results for stimulated (DCCT), fasting (CACTI and EDC) and random (Joslin 50-Year Medalist and WESDR) C-peptide (Fig. 1). We specifically examined the associations of known type 1 diabetes loci and type 1 diabetes genetic risk scores (GRS) with C-peptide levels [14, 17]. The associations of the identified loci and type 1 diabetes loci/GRS were tested with stimulated C-peptide change over time in a subset of DCCT participants with repeated annual measures for up to 6 years $(n=258)$.

\section{Studies}

DCCT Individuals aged 13-39 years with type 1 diabetes and fasting serum C-peptide levels $\leq 0.2 \mathrm{pmol} / \mathrm{ml}$ were recruited between 1983 and 1989. The primary cohort included participants with 1-5 years of diabetes, no pre-existing retinopathy, normal albuminuria ( $<40 \mathrm{mg} / 24 \mathrm{~h}$ ) and stimulated C-peptide $\leq 0.5 \mathrm{pmol} / \mathrm{ml}$. The secondary cohort included participants with 1-15 years of diabetes, pre-existing mild retinopathy, albumin excretion rate $<200 \mathrm{mg} / 24 \mathrm{~h}$ and stimulated Cpeptide $\leq 0.5$ and $0.2 \mathrm{pmol} / \mathrm{ml}$ for participants with diabetes duration 1-5 and 5-15 years, respectively [ESM Table 3]. Participants were randomly assigned to receive intensive or conventional insulin treatment and were monitored until 1993 [18]. C-peptide levels were measured annually for up to 6 years in those with stimulated C-peptide $>0.2 \mathrm{pmol} / \mathrm{ml}$ at eligibility testing $[19,20]$. 


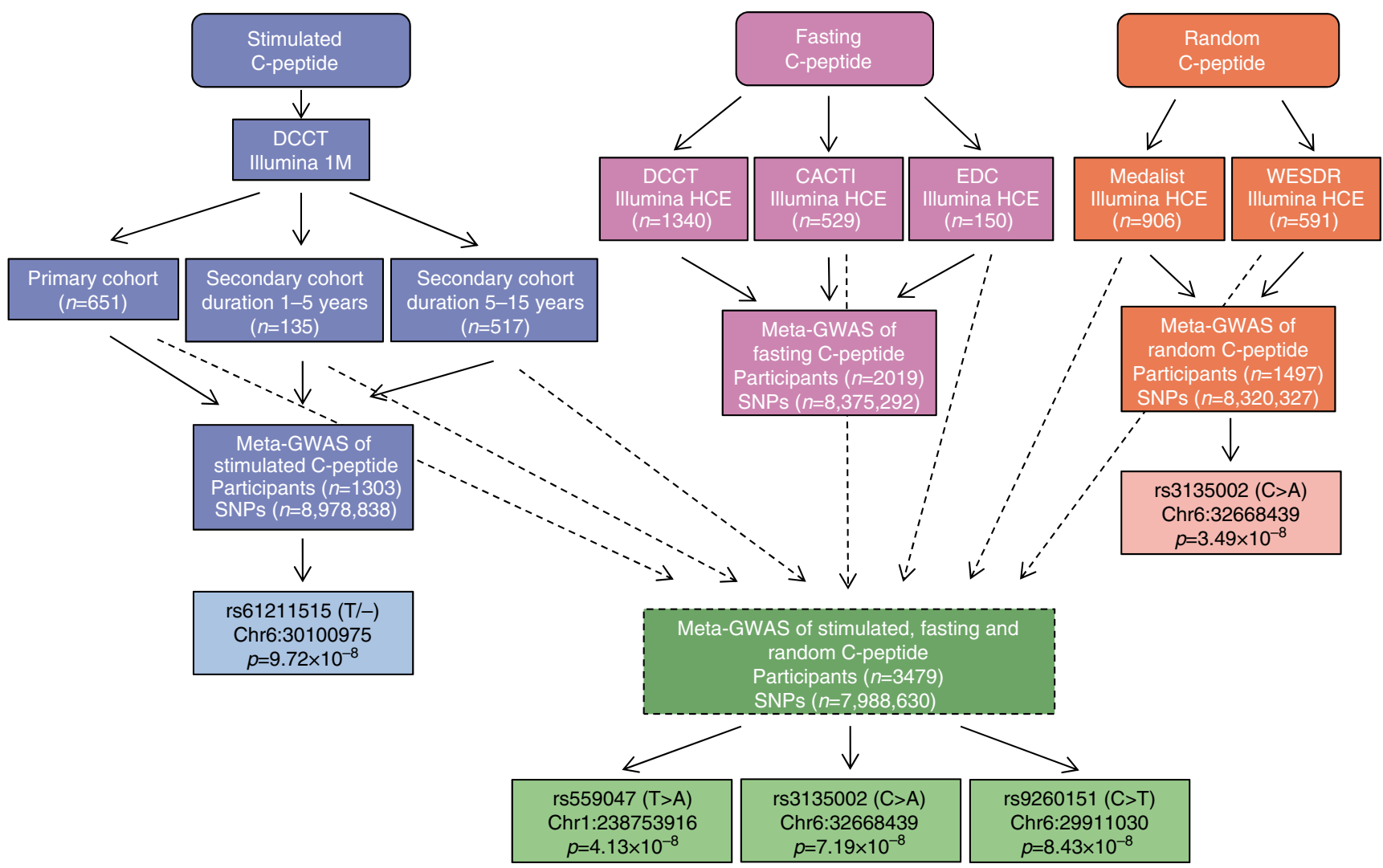

Fig. 1 Overview of the C-peptide cross-sectional data analysis and the main findings. The dashed arrows show the studies included in the meta-GWAS of stimulated, fasting and random C-peptide. HCE, human core exome

CACTI CACTI was an observational, population-based study of individuals with type 1 diabetes of duration $>10$ years at enrolment. Participants were recruited from Denver, CO, USA, between March 2000 and April 2002 [21]. Fasting serum Cpeptide was measured during 2014-2016 [22] ESM Table 3].

EDC This study recruited a cohort of individuals with incident, childhood-onset ( $<17$ years of age) type 1 diabetes who were diagnosed or seen within 1 year of diagnosis (1950-1980) at the Children's Hospital of Pittsburgh. The first participant assessments occurred in 1986-1988 [23]. Serum C-peptide was measured at follow-up year 25 [ESM Table 3]. Participants were advised to fast for $\geq 8 \mathrm{~h}$, but $17 \%(n=26)$ did not. Of the participants who failed to fast, only $15 \%(n=4)$ had detectable C-peptide (unpublished data, T. J. Orchard and T. Costacou).

Joslin 50-year Medalist study This was a cross-sectional study. All participants provided their original medical record or three forms of documentation of $\geq 50$ years of insulin-dependent diabetes and also resided in the USA at the time of participation [24]. Random serum C-peptide levels were measured and validated at the Northwest Lipid Research Laboratory at the University of Washington [ESM Table 3] [13].

WESDR People with diabetes who received primary care in an 11-county area in southern Wisconsin from 1979 to 1980 were recruited and subsequently followed for diabetic complications $[25,26]$. The participants were all younger-onset individuals who were diagnosed before 32 years of age and treated with insulin. Random serum C-peptide levels were measured at years 4 and 10 of follow-up [ESM Table 3] [27], with the most recent results used for analysis.

\section{Genotyping and imputation}

Genotyping was performed using Illumina 1M [28] and HumanCoreExome BeadArrays (Illumina, San Diego, CA, USA) in the DCCT. Illumina HumanCoreExome BeadArrays were used for genotyping in the CACTI, EDC, Joslin 50-Year Medalist and WESDR studies. Ungenotyped autosomal SNPs were imputed using 1000 Genomes data (phase 3, v5) (see ESM Methods for more details) [29].

Genotype dosage data from Illumina 1M BeadArrays were used to analyse stimulated C-peptide in the DCCT, as approximately 3 million more SNPs were imputed with good quality (INFO $\geq 0.8$ ); and genotype dosage data from HumanCoreExome BeadArrays were used for the remaining analyses in all studies. SNPs with minor allele frequency (MAF) $>0.01$ and high imputation quality (INFO $>0.8$ and $R^{2}>0.5$ from IMPUTE2 and Minimac, respectively; see ESM Methods for software details) were included in the analysis. 


\section{GWAS and meta-GWAS of cross-sectional C-peptide}

SNPs were tested for association with C-peptide (natural log transformed) under an additive genetic model using Tobit models to appropriately model the large number of participants with values at the lower limit of detection (ESM Table 3) [30]. The Tobit model beta coefficient can be interpreted as the relationship between the genotype and the uncensored latent (unobserved) C-peptide variable. VGLM models from the VGAM library v0.9-7 were fitted using R v3.1.0 [31]. Sex, age at diagnosis and diabetes duration were included as covariates in the model. For fasting C-peptide analysis in the DCCT, the cohort (primary vs secondary) was also included as a covariate in the model.

Meta-GWAS analysis of stimulated C-peptide was performed using METAL v1.5 (www.sph.umich.edu/csg/ abecasis/Metal/index.html) with the STDERR method, which weights effect-size estimates by the inverse of the corresponding SEs. However, because C-peptide was measured using different assays in the different studies, and effect-size estimates and standard errors were in different units, the other meta-GWAS analyses used the SAMPLESIZE method, which converts the SNP association test $p$ values to $Z$ scores using the direction of effect, and combines $Z$ scores across studies weighting by the study-specific sample size. Between-study heterogeneity was tested using Cochran's $Q$ statistic. $I^{2}$, which depends on $Q$, is used to quantity the level of heterogeneity among studies [32].

\section{Testing for the heterogeneity of the SNP effect between stimulated and fasting C-peptide}

To investigate whether the SNP associations differed between stimulated and fasting C-peptide in the DCCT, we used the Tobit model implementation in the Zelig v 4.2-1 library in R v3.1.0 [31] to account for within-individual correlations of the two C-peptide measurements. Sex, age at diagnosis, diabetes duration, cohort (primary vs secondary) and an indicator for fasting/stimulated C-peptide were included as covariates in the models. Heterogeneity was tested using an interaction between the fasting/stimulated indicator and SNP.

\section{Type 1 diabetes loci}

We investigated two sets of type 1 diabetes loci derived from Oram et al [17] and Onengut-Gumuscu et al [14]. The study of Oram et al included robustly associated genetic variants in both MHC and non-MHC regions from published studies ( $n=30$ SNPs) [33-36], whereas Onengut-Gumuscu et al included only non-MHC regions and was based on Immunochip data in $>9000$ participants with type 1 diabetes and $>12,000$ non-diabetic control participants with multiple independent variants in some loci $(n=51 \mathrm{SNPs}$ ) (see ESM Methods for details).

We stratified SNPs according to the presence ( $n=70$ SNPs) or absence of a prior association with type 1 diabetes, and performed false discovery rate (FDR) control separately for each stratum using the stratified (S)FDR method [37, 38].

\section{MHC imputation}

Classical HLA alleles, their amino acid sequences and additional SNPs in the MHC region were imputed using genotyped SNPs from Illumina 1M BeadArrays in DCCT and Illumina HumanCoreExome BeadArrays in the Joslin 50Year Medalist, CACTI, WESDR and EDC studies; and SNP2HLA (http://software.broadinstitute.org/mpg/snp2hla). The Type 1 Diabetes Genetic Consortium (T1DGC) dataset (5196 unrelated individuals, including 4323 European participants: 182 participants with type 1 diabetes, 4101 control participants and 182 participants with missing case/control status [39]) was used as the reference panel. The T1DGC panel contains 5868 SNPs (genotyped using the Illumina Immunochip) and four-digit classical HLA types (HLA-A, $B,-C,-D P A 1,-D P B 1,-D Q A 1,-D Q B 1$ and $-D R B 1$ ) [40].

Each of the classical HLA alleles, their amino acid sequences and SNPs in the MHC region were tested for associations with $\mathrm{C}$-peptide, and combined through meta-analysis (as described above).

\section{Longitudinal stimulated C-peptide in the DCCT}

Associations of identified SNPs with repeated measures of stimulated C-peptide (natural log transformed) were tested under an additive genetic model using linear mixed models (random slope and random intercept) and first-order autoregressive AR(1) correlation structure in R v3.1.0 [31]. SNP, sex, age at diagnosis, diabetes duration at baseline, treatment group (intensive vs conventional), cohort (primary vs secondary), an indicator for baseline (vs later assessment), time (DCCT follow-up year), (centred time) ${ }^{2}$; and interactions between time and treatment, treatment and baseline, and SNP and time were included in the model. Differences among SNP genotypes in the rate of C-peptide decline over time were investigated by hypothesis testing of SNP and time interaction.

\section{Results}

\section{Meta-GWAS}

Characteristics of the participants included in stimulated ( $n=$ 1303), fasting ( $n=2019)$, random $(n=1497)$, and stimulated/ fasting/random $(n=3479)$ C-peptide meta-GWAS are 
Table 1 Association of rs559047 (Chr1: 238753916, T>A) with C-peptide in different studies

\begin{tabular}{|c|c|c|c|c|c|c|c|}
\hline Analysis/study & $\mathrm{INFO} / R^{2}$ & MAF & $\beta$ & $\mathrm{SE}$ & $p$ & Het $I^{2}$ & Het $p$ \\
\hline \multicolumn{8}{|l|}{ Stimulated C-peptide } \\
\hline DCCT, primary & 0.98 & 0.24 & -0.25 & 0.07 & $8.87 \times 10^{-4}$ & & \\
\hline DCCT, secondary (duration $1-5$ years) & 0.98 & 0.24 & -0.44 & 0.16 & 0.007 & & \\
\hline DCCT, secondary (duration 5-15 years) & 0.98 & 0.24 & -0.27 & 0.13 & 0.041 & & \\
\hline Meta-GWAS & & & -0.28 & 0.06 & $3.74 \times 10^{-6}$ & 1.18 & 0.55 \\
\hline \multicolumn{8}{|l|}{ Fasting C-peptide } \\
\hline DCCT & 0.96 & 0.23 & -0.13 & 0.05 & 0.014 & & \\
\hline CACTI & 0.95 & 0.24 & -0.6 & 0.35 & 0.092 & & \\
\hline EDC & 0.96 & 0.25 & -1.04 & 0.86 & 0.227 & & \\
\hline Meta-GWAS & & & & & $1.36 \times 10^{-3}$ & 0 & 0.94 \\
\hline \multicolumn{8}{|l|}{ Random C-peptide } \\
\hline Joslin 50-Year Medalist & 0.95 & 0.25 & -0.09 & 0.04 & 0.029 & & \\
\hline WESDR & 0.96 & 0.26 & -0.62 & 0.36 & 0.088 & & \\
\hline Meta-GWAS & & & & & $5.60 \times 10^{-3}$ & 0 & 0.97 \\
\hline $\begin{array}{l}\text { Stimulated/fasting/random } \\
\text { C-peptide meta-GWAS }\end{array}$ & & & & & $4.13 \times 10^{-8}$ & 0 & 0.61 \\
\hline
\end{tabular}

$\mathrm{INFO} / R^{2}$ indicates the quality of imputation

Alleles are non-effect allele $>$ effect allele

C-peptide was measured with different assays in pmol/ml in the DCCT, Joslin 50-Year Medalist study and WESDR; and in pmol/l in the CACTI and EDC studies; it was also natural log transformed

$\beta, \beta$ coefficient; Het, heterogeneity

summarised in ESM Tables 4, 5. C-peptide distributions in different studies are shown in ESM Figs 1, 2.

1q53 In the stimulated/fasting/random C-peptide metaGWAS, rs559047 on 1q53 (Chr1: 238753916, T>A) exceeded the genome-wide significance threshold $(p<5 \times$ $10^{-8}$ )(Fig. 1; ESM Figs 3, 4). The A allele was associated with lower C-peptide $\left(p=4.13 \times 10^{-8}\right)$. The direction of the rs559047 association with $\mathrm{C}$-peptide was consistent in all studies (Table 1, Fig. 2).

At DCCT eligibility, fasting and stimulated C-peptide levels were highly positively correlated (Spearman correlation coefficient $0.88, p<0.0001)$. rs559047 was associated with both stimulated and fasting C-peptide at DCCT eligibility, but there was a significant difference in the association between stimulated and fasting C-peptide $(p=0.010)$, with a larger effect on stimulated compared with fasting C-peptide $(-0.28$ vs -0.13$)$. The association of rs 559047 with stimulated $\mathrm{C}$-peptide remained significant after including fasting $\mathrm{C}$ peptide in the model but not vice versa, suggesting that the rs559047 association with fasting C-peptide is indirect and due to its association with stimulated C-peptide (Table 2).

rs559047 was not associated with the rate of decline in stimulated C-peptide over time using longitudinal DCCT data. Characteristics of the participants included in the DCCT longitudinal data analysis are summarised in ESM Table 6.
MHC region Multiple variants in the $\mathrm{MHC}$ region were associated with different C-peptide measures. In the stimulated/ fasting/random C-peptide meta-GWAS, two loci in the MHC region, rs3135002 (Chr6:32668439) and rs9260151 (Chr6:29911030), approximately $2.8 \mathrm{Mb}$ apart from each other, were close to genome-wide significance. A single nucleotide deletion in the MHC region, rs61211515 (Chr6:30100975), had the lowest $p$ value in the stimulated C-peptide meta-GWAS (Fig. 1; ESM Figs 3, 4). Detailed results for these three loci follow.

rs3135002 rs3135002 (C>A) exceeded the genome-wide significance threshold in the random C-peptide meta-GWAS ( $p=$ $3.49 \times 10^{-8}$ ). The A allele was associated with higher random C-peptide in both the Joslin 50-Year Medalist study $(\beta[\mathrm{SE}]=$ $\left.0.44[0.12], p=3.22 \times 10^{-4}\right)$ and the WESDR $(\beta[\mathrm{SE}]=2.34$ [0.54], $\left.p=1.53 \times 10^{-5}\right)$. rs3135002 was also associated with fasting C-peptide at DCCT eligibility $(\beta[\mathrm{SE}]=0.36[0.13]$, $\left.p=5.42 \times 10^{-3}\right)$. Although it was not associated with stimulated C-peptide in the DCCT ( $\beta[\mathrm{SE}]=0.27[0.15], p=0.077)$, the effect was in the same direction; further, there was no significant difference between the fasting and stimulated Cpeptide associations $(p=0.46)$. rs 3135002 was not associated with fasting C-peptide in the CACTI or EDC studies, and nor was it associated with the rate of stimulated C-peptide decline over time in the DCCT ( $p=0.62)$ (Table 3, Fig. 3). 


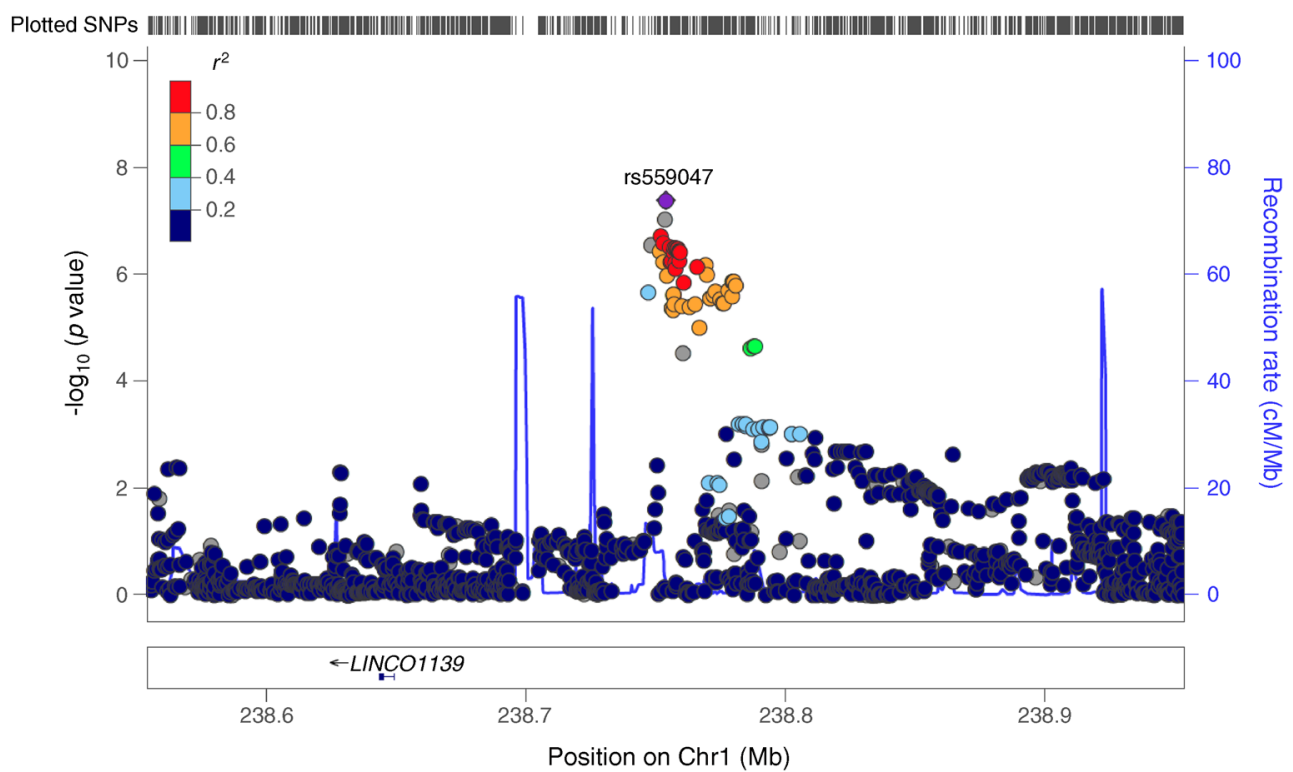

Fig. 2 Stimulated/fasting/random C-peptide meta-GWAS, which includes stimulated C-peptide results from the DCCT primary cohort, the secondary cohort with diabetes duration 1-5 years and the secondary cohort with diabetes duration 5-15 years; fasting C-peptide results from the CACTI and EDC studies; and random C-peptide results from the Joslin 50-Year Medalist study and WESDR. The left $y$-axis shows SNP $p$ values from the stimulated/fasting/random C-peptide meta-GWAS. The right $y$-axis shows estimated recombination rates. The $x$-axis shows SNP genomic position (Genome Reference Consortium Human Build 37 [GRCh37]/hg19); the LD measures are based on 1000 Genomes Nov 2014 EUR population. The plot was created using LocusZoom (http://locuszoom.sph.umich.edu/locuszoom) [44]. Chr, chromosome rs9260151 rs9260151 (C>T) is an intronic SNP within HLA$A$. Its $\mathrm{T}$ allele was associated with higher $\mathrm{C}$-peptide with a $p$ value close to the genome-wide significance threshold in the stimulated/fasting/random C-peptide meta-GWAS ( $p=$ $\left.8.43 \times 10^{-8}\right)$. The direction of the effect was consistent in all studies (Table 3, Fig. 3). rs9260151 was associated with both stimulated and fasting C-peptide at DCCT eligibility but the effects were significantly different $(p=0.024)$, with a larger effect on stimulated compared with fasting C-peptide ( $0.34 \mathrm{vs}$ $0.20)$. Its association with stimulated $\mathrm{C}$-peptide remained significant after including fasting C-peptide in the model, but not vice versa (Table 2). rs9260151 was not associated with differences in the rate of decline in stimulated C-peptide over time using longitudinal DCCT data.

rs61211515 A single nucleotide deletion in the $\mathrm{MHC}$ region, rs61211515 (T/ -$)$, had the lowest $p$ value $(\beta[\mathrm{SE}]=-0.39$ [0.07], $p=9.72 \times 10^{-8}$ ) in the stimulated C-peptide metaGWAS (Table 3, Fig. 3). rs61211515 was also associated with fasting C-peptide at DCCT eligibility $(\beta[\mathrm{SE}]=-0.23[0.07]$, $\left.p=6.80 \times 10^{-4}\right)$. The association of rs61211515 (T/-) with stimulated C-peptide remained significant after including fasting $\mathrm{C}$-peptide in the model $(\beta[\mathrm{SE}]=-0.28[0.05], p=$ $2.77 \times 10^{-8}$ ), whereas its association with fasting $\mathrm{C}$-peptide became insignificant after including stimulated $\mathrm{C}$-peptide in the model $(p=0.10)$ (Table 2). The strength of rs61211515 association with fasting and stimulated C-peptide differed significantly $\left(p=4.66 \times 10^{-3}\right)$, with a larger effect on stimulated compared with fasting C-peptide $(-0.39$ vs -0.23$)$. rs61211515 (T/-) was not significantly associated with fasting C-peptide in the CACTI or EDC studies, but it was associated with random $C$-peptide in WESDR with the same direction of effect $(\beta[\mathrm{SE}]=-0.94[0.45], p=0.035)$. Although its association with random $\mathrm{C}$-peptide was not significant in the Joslin 50 -Year Medalist study $(\beta[\mathrm{SE}]=-0.10[0.05], p=0.065)$, the effect direction was the same (Table 3). The rate of decline in stimulated C-peptide over time in the DCCT depended on the rs61211515 (T/-) genotype (SNP-time interaction $\beta[\mathrm{SE}]=$ -0.08 [0.04], $p=0.023$ ). Association of rs61211515 with stimulated C-peptide at DCCT eligibility in participants with and without longitudinal data is shown in ESM Table 7. rs61211515 is a mononucleotide T repeat $(n=12$ Ts in RefSeq (https://www.ncbi.nlm.nih.gov/refseq/, accessed 1 July, 2017)). An insertion allele at the same locus (T/TT) was not associated with stimulated, fasting or random Cpeptide; and comparing five genotype categories with a homozygous reference, only categories with deletion had significant effects (ESM Tables 8, 9).

Independence of the MHC signals rs9260151, rs3135002 and rs61211515 are in linkage disequilibrium (LD) (ESM Table 10). However, putting them in the same model, all three remained significantly associated with stimulated and fasting C-peptide at DCCT eligibility (ESM Table 11). The likelihood ratio test comparing a model with no SNPs (only covariates in the model and stimulated C-peptide as the outcome) to a model with all three SNPs (rs61211515, rs9260151, rs3135002 and covariates) was strongly significant $\left(p=5.67 \times 10^{-10}\right)$. 


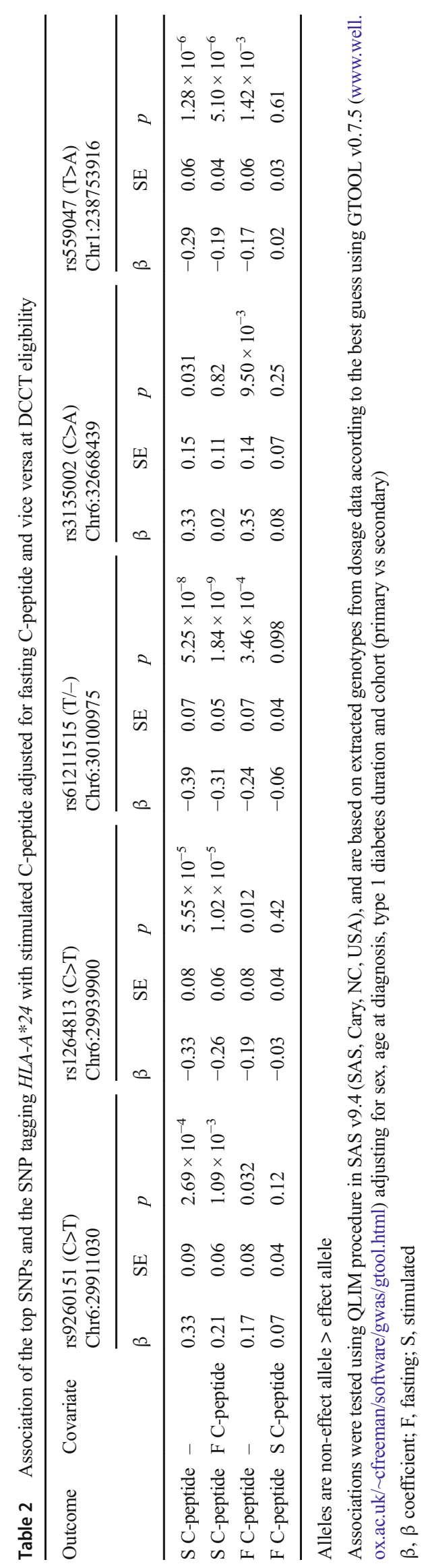




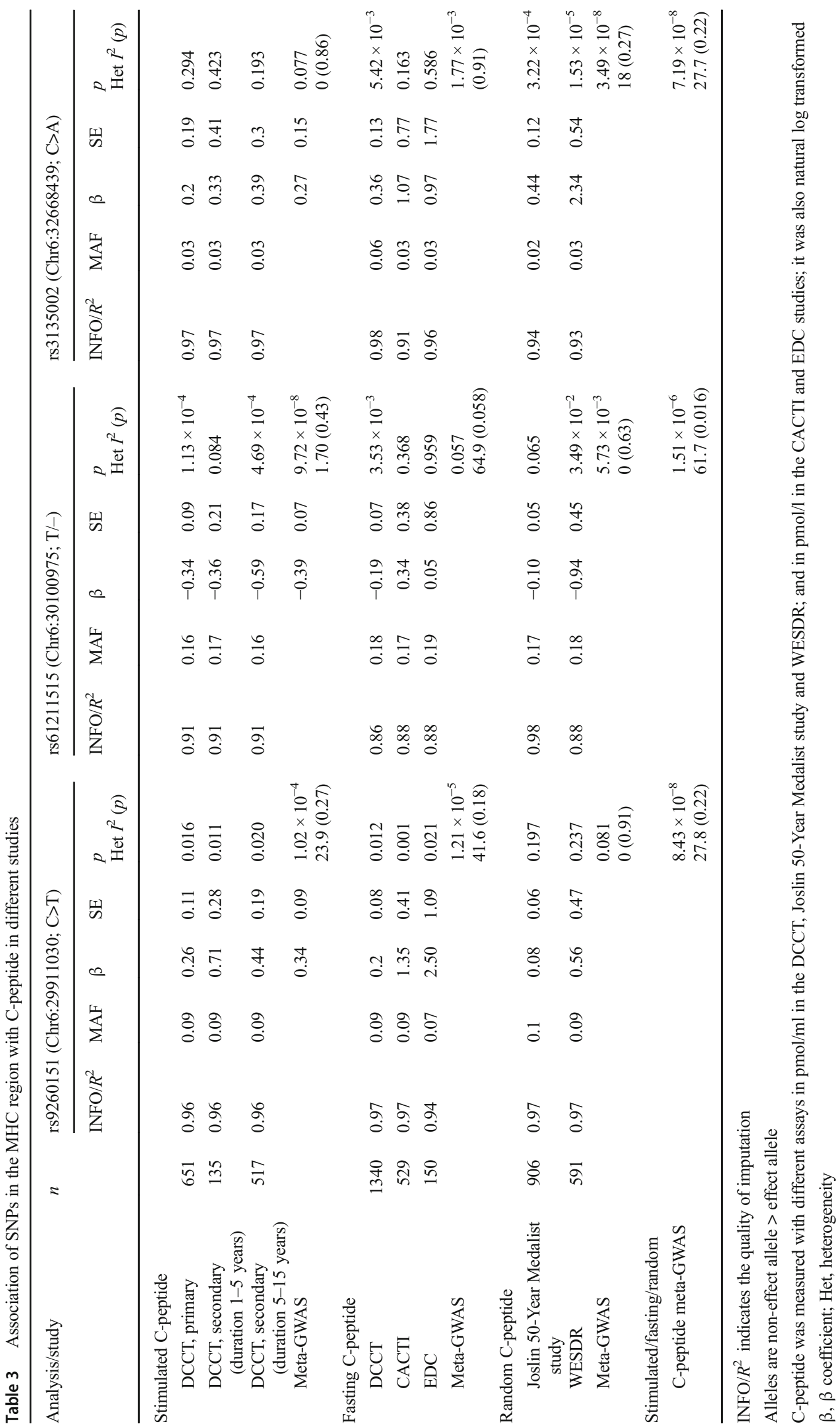


a

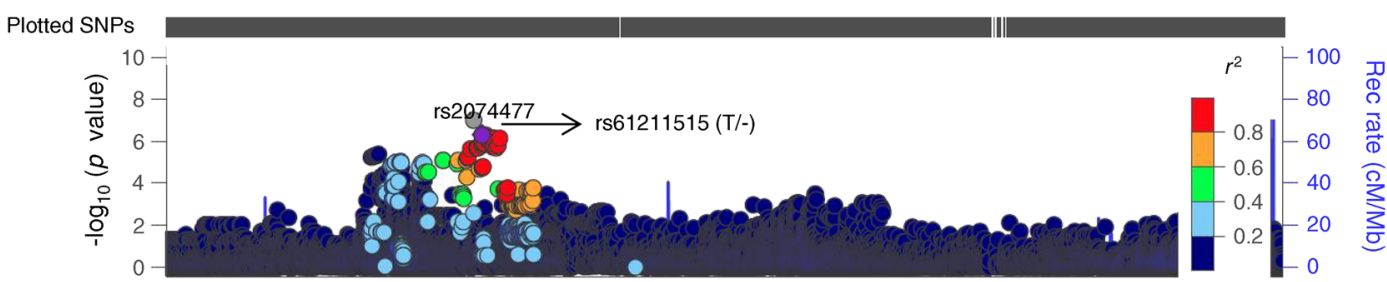

b

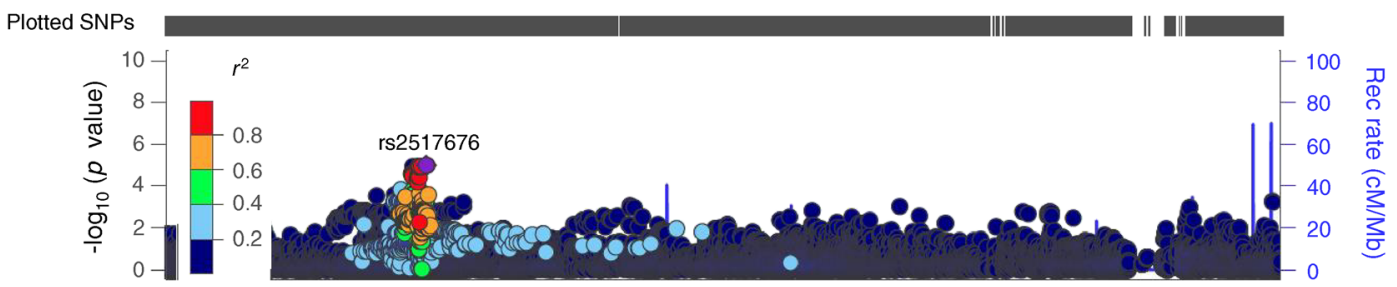

C

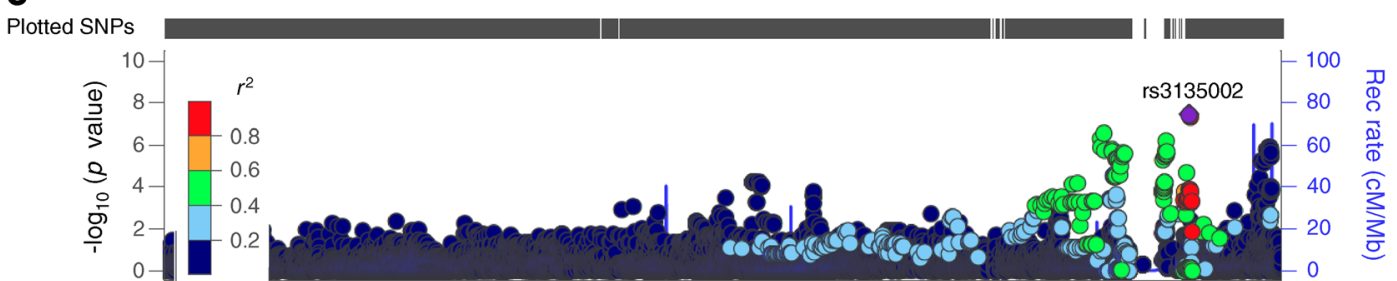

d
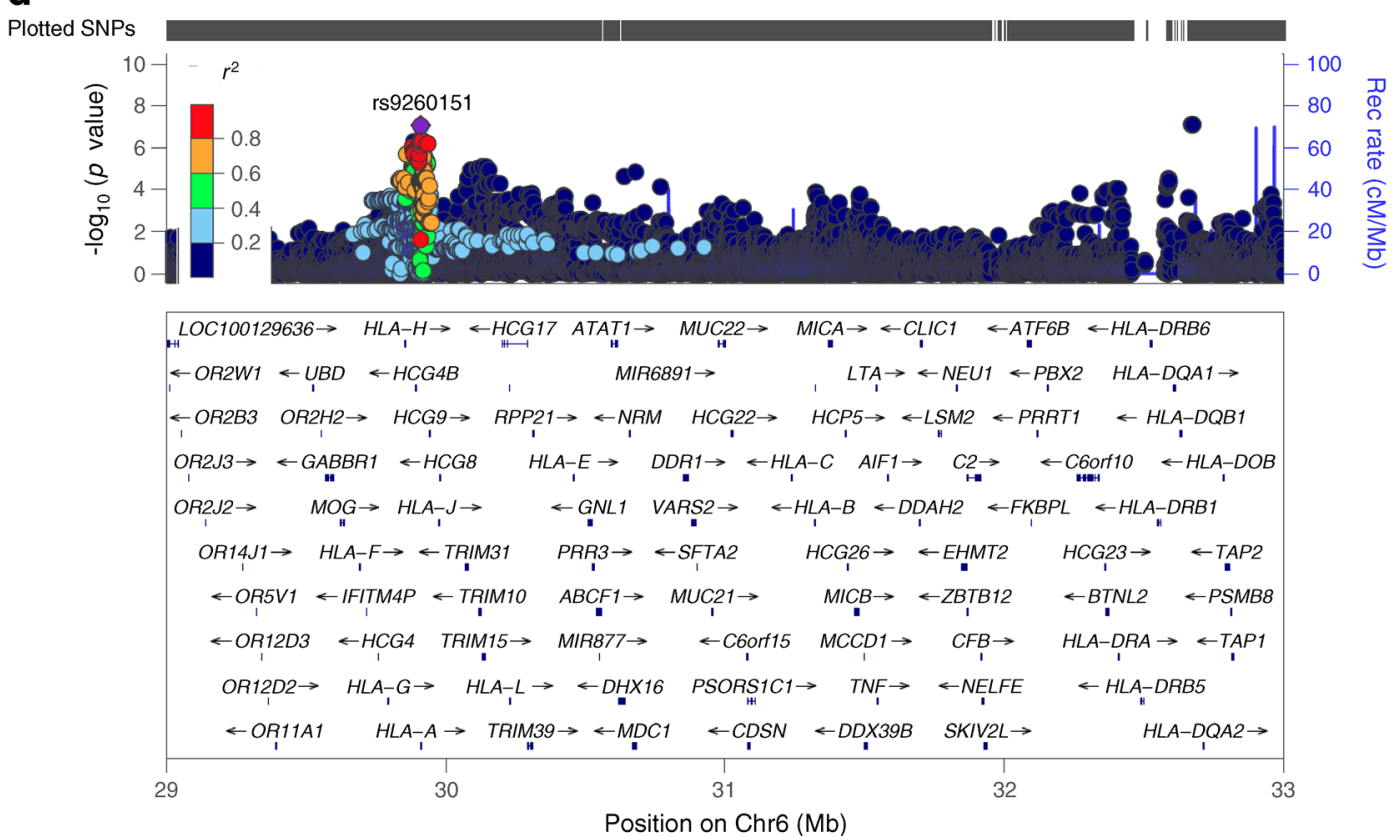

Fig. 3 (a) Stimulated C-peptide meta-GWAS, which includes the DCCT primary cohort, the secondary cohort with diabetes duration 15 years and the secondary cohort with diabetes duration $5-15$ years. Since rs61211515 (T/-) is an insertion/deletion (in/del) second-to-top hit, rs 2074477, in high LD with rs61211515 $\left(r^{2} 0.89 ; D^{\prime} 0.98\right)$ was used by LocusZoom for LD measures. (b) Fasting C-peptide meta-GWAS, which includes the DCCT, CACTI and EDC studies. (c) Random C-peptide meta-GWAS, which includes the Joslin 50-Year Medalist study and WESDR. (d) Stimulated/fasting/random C-peptide meta-GWAS, which includes stimulated C-peptide results from the DCCT primary cohort, the secondary cohort with diabetes duration $1-5$ years and the secondary cohort with diabetes duration 5-15 years; fasting C-peptide results from the CACTI and EDC studies; and random C-peptide results from the Joslin 50-Year Medalist study and WESDR. Left $y$-axis: SNP $p$ values; right $y$-axis: estimated recombination rates; $x$-axis: SNP genomic position (Genome Reference Consortium Human Build 37 [GRCh37]/hg19); the LD measures are based on the 1000 Genomes November 2014 EUR population. The plot was made using LocusZoom (http://locuszoom. sph.umich.edu/locuszoom/) [44]. Chr, chromosome; Rec, recombination 
MHC imputation A total of 424 classical HLA alleles were imputed: 279 were polymorphic, and of them 252 had good imputation quality $\left(R^{2}>0.5\right)$ in the DCCT. Similarly, $1136 / 1276$ amino acid changes were polymorphic, and 1106 met imputation quality criteria. A total of 7144/7261 SNPs were polymorphic and 7066 were imputed well (ESM Table 12). None of the HLA classical alleles or amino acid changes reached the genome-wide significant threshold in the meta-GWAS.

\section{Association of type 1 diabetes loci and C-peptide}

Five type 1 diabetes risk loci were nominally associated $(p<0.05)$ with lower stimulated C-peptide at DCCT eligibility: rs1264813 tagging $H L A-A * 24$ ( $\beta$ [SE] $=-0.34$ [0.08], $p=$ $\left.2.80 \times 10^{-5}\right), \operatorname{rs} 151234($ IL27) $(\beta[\mathrm{SE}]=-0.32[0.08], p=$ $\left.3.15 \times 10^{-5}\right), \operatorname{rs} 12971201(P T P N 2)(\beta[\mathrm{SE}]=-0.13[0.05]$, $\left.p=9.38 \times 10^{-3}\right), \operatorname{rs} 689($ INS $)(\beta[\mathrm{SE}]=-0.18[0.07], p=$ $0.011)$, and rs193778 (DEXI) $(\beta[\mathrm{SE}]=-0.11[0.06], p=$ 0.044). Association of these SNPs with fasting/random Cpeptide in the other studies is shown in ESM Table 13.

The SFDR method estimated the proportion of null SNPs $\left(\pi_{0}\right)$ at 0.91 in the prior type 1 diabetes stratum vs 0.99 in the stratum of the remaining SNPs. Setting an FDR control level of $20 \%$ yielded four test rejections for prior type 1 diabetes SNPs and no test rejections in the remaining SNPs. The FDR $q$ value estimates were 0.001 for both rs1264813 and rs151234, and 0.17 for both rs12971201 and rs689. SFDR ranked these four SNPs above rs61211515, the top associated SNP in the stimulated C-peptide meta-GWAS.

The association of rs1264813 (HLA-A*24) with stimulated C-peptide at DCCT eligibility did not remain significant when rs61211515 (the top GWAS-associated SNP in this region) was included in the model $(p=0.45)$. Similar results were observed with imputed $H L A-A * 24$; it was associated with lower stimulated C-peptide at DCCT eligibility $(\beta[\mathrm{SE}]=-0.39[0.09], p=$ $\left.1.50 \times 10^{-5}\right)$, but this association became insignificant after adjustment for rs61211515 ( $p=0.09$ ) (ESM Table 14). rs1264813 $(H L A-A * 24)$ was also nominally associated with fasting Cpeptide at DCCT eligibility $(\beta[\mathrm{SE}]=-0.19$ [0.07], $p=0.013$ ) but its association with stimulated C-peptide was significantly stronger $(p=0.011)$. The rs1264813 association with stimulated C-peptide remained significant when fasting C-peptide was included in the model but not vice versa (Table 2). rs1264813 was also associated with an increased rate of decline in stimulated C-peptide over time in the DCCT (SNP-time interaction $\left.\beta[\mathrm{SE}]=-0.11[0.04], p=6.32 \times 10^{-3}\right)$.

$H L A-D R 3(\beta[\mathrm{SE}]=0.03[0.06], p=0.59)$ and HLA-DR4$D Q 8(\beta[\mathrm{SE}]=0.06[0.06], p=0.29)$ were not associated with stimulated C-peptide at DCCT eligibility. $H L A-D R 3 / D R 4-D Q 8$ compound heterozygotes had higher stimulated C-peptide compared with individuals having no copy of $D R 3$ or $D R 4-D Q 8$ ( $\beta$ $[\mathrm{SE}]=0.26[0.11], p=0.024)$. However, this association did not withstand multiple testing correction (ESM Table 15).
HLA-DQB1 position 57, HLA-DRB1 position 13 or $H L A$ $D R B 1$ position 71 amino acid variants, which have been reported to independently drive type 1 diabetes risk and explain $>90 \%$ of the type 1 diabetes-HLA association [41], were not associated with stimulated C-peptide at DCCT eligibility.

Of the two type 1 diabetes GRS, only the OnengutGumuscu et al type 1 diabetes GRS was nominally associated with lower stimulated C-peptide $(\beta[\mathrm{SE}]=-0.09$ [0.04], $p=$ $0.036)$ at DCCT eligibility. This association did not remain significant after excluding rs151234 (IL27); $\beta[\mathrm{SE}]=-0.07$ [0.04], $p=0.085$.

\section{Discussion}

By combining GWAS data from five type 1 diabetes studies including approximately 1300 individuals with stimulated and approximately 3500 individuals with stimulated, fasting or random C-peptide data, we identified association with a locus on chromosome 1 (rs559047) and multiple independent variants in the MHC region.

rs559047 is an intergenic SNP located $700 \mathrm{~kb}$ from the nearest gene (ZP4). It is not a significant cis-expression quantitative trait locus (cis-eQTL) for nearby genes in any tissue, including the pancreas (www.gtexportal.org, accessed 14 July 2017) or pancreatic islets (http://theparkerlab.org/tools/isleteqtl, accessed 14 July 2017). However, the cis-eQTLs were produced using a limited number of samples (149 and 112 for pancreas and islets, respectively) from non-diabetic individuals. rs559047 has not been associated with the risk of type 1 diabetes (ESM Table 16), and variants in this region have not previously been associated with type 1 diabetes related phenotypes.

We identified three SNPs (rs9260151, rs61211515 and rs3135002) in the MHC region associated with C-peptide levels. Of these, rs9260151 and rs3135002 have been associated with risk of type 1 diabetes (ESM Table 16). rs9260151 is an intronic SNP within $H L A-A$, while rs61211515 is located approximately $187 \mathrm{~kb}$ downstream of HLA-A. Both SNPs appear to be primarily associated with stimulated C-peptide although rs9260151 was identified in the meta-GWAS of stimulated/fasting/random C-peptide, likely due to the larger available sample size. HLA$A * 24$ is a known risk variant for type 1 diabetes [34, 42]. However, after including rs9260151, rs61211515 and HLA$A * 24$ (either imputed or tagging SNP) in the model, rs9260151 and rs61211515 both remained significantly associated with $\mathrm{C}$-peptide, indicating that they are independent signals and distinct from $H L A-A * 24$. The association between $H L A-$ $A * 24$ and C-peptide was no longer significant, suggesting that this association is due to LD between $H L A-A * 24$ and rs9260151 and/or rs61211515 (ESM Table 10). Similarly, the association of rs9260151 with type 1 diabetes could be explained by its LD with $H L A-A * 24$. rs61211515 and HLA-A *24, which were associated with lower $\mathrm{C}$-peptide levels, were also associated with an 
accelerated decline in C-peptide levels over time, providing more evidence that this association is a true finding. HLA$A * 24$ has previously been associated with undetectable Cpeptide in a Japanese type 1 diabetes population sample [15] and with more rapid beta cell destruction post islet transplantation [43]. The third SNP, rs3135002, is intergenic between HLA$D Q B 1$ and $D Q A 2,2.5 \mathrm{Mb}$ from rs9260151 and rs61211515. Its association with C-peptide remained significant when both rs9260151 and rs61211515 were included in the model, indicating that it is independent. $D R 3$ and $D R 4-D Q 8$ were not associated with $\mathrm{C}$-peptide and the borderline association observed between $D R 3 / D R 4-D Q 8$ compound heterozygotes and Cpeptide did not withstand multiple testing correction, suggesting that this effect is also distinct from the DR3/DR4-DQ8 haplotype, consistent with previous findings [15]. The association of rs3135002 with type 1 diabetes could be explained by the extremely large effect of $D R 3 / D R 4-D Q 8$ on type 1 diabetes and the LD between rs3135002 and both $D R 3\left(D^{\prime}=1, r^{2}=0.012\right)$ and $D R 4-D Q 8\left(D^{\prime}=0.87, r^{2}=0.010\right)$. The rs3135002 association was mainly with fasting/random C-peptide rather than with stimulated C-peptide. No significant interactions were observed between the three SNPs in the MHC affecting stimulated/fasting C-peptide (data not shown), and none of them was a significant cis-eQTL for the nearby genes in any tissue including the pancreas and pancreatic islets (http://www.gtexportal.org, accessed July 14, 2017; http://theparkerlab.org/tools/isleteqtl, accessed July 14, 2017).

Some SNP associations for C-peptide in type 1 diabetes are different from those for type 1 diabetes. Two of the identified variants (rs559047 on chromosome 1 and rs61211515 in the MHC region) have not been previously associated with type 1 diabetes; while rs9260151 and rs3135002 in the MHC region have been associated with type 1 diabetes. The variants associated with C-peptide but not with type 1 diabetes may affect gene expression, and fine-mapping and functional studies are required to determine their effects. However, SFDR analysis suggests that the proportion of type 1 diabetes loci associated with C-peptide is greater than for the rest of the genome; four out of 70 type 1 diabetes loci (HLA-A*24, IL27, INS and PTPN2) that were nominally associated with lower C-peptide levels ranked higher than rs61211515, the top associated SNP in the stimulated C-peptide meta-GWAS. Nevertheless, the composition and magnitude of SNP effects for type 1 diabetes development vs progression could differ, explaining why there is no significant association between type 1 diabetes GRS and C-peptide.

Some limitations include that only fasting/random C-peptide measurements were available for about $60 \%$ of individuals, whereas stimulated C-peptide is more appropriate to evaluate insulin secretion in type 1 diabetes. In addition, individuals from different cohorts were quite different in terms of their age at diagnosis, diabetes duration and other inclusion/exclusion criteria, all of which may influence C-peptide levels. C-peptide can also be measured with different methods with different lower limits of detection, and these were not standardised among the studies. The mean age at diagnosis ranged from 8.3 years in the EDC study to 21.2 years in the DCCT, and the mean type 1 diabetes duration ranged from 5.6 years in the DCCT to 54.7 years in the Joslin 50-Year Medalist study. Participants from the DCCT, the only study with stimulated C-peptide data, were highly selected as they were diagnosed with type 1 diabetes later in life and had diabetes for a shorter period of time. Therefore, the DCCT had a larger proportion of participants with detectable Cpeptide compared with the other studies. This heterogeneity among the cohorts might have reduced the power of the analysis and contributed to non-replication at specific loci.

In conclusion, we have identified a locus on chromosome 1 and multiple variants in the MHC region that are associated with C-peptide levels. However, these associations need to be validated in independent populations. Further investigations could eventually provide insight into mechanisms of beta cell loss and opportunities to preserve beta cell function.

Acknowledgements The authors acknowledge the participants and researchers of the DCCT/EDIC, Joslin 50-Year Medalist, CACTI, WESDR and EDC studies. This research used resources provided by the T1DGC, a collaborative clinical study sponsored by the National Institute of Diabetes and Digestive and Kidney Diseases (NIDDK) (U01-DK062418), National Institute of Allergy and Infectious Diseases, National Human Genome Research Institute, National Institute of Child Health and Human Development, and JDRF International. The authors acknowledge C. C. Robertson and S. S. Rich (Center of Public Health Genomics, University of Virginia, Charlottesville, VA, USA) for providing the descriptive data regarding the T1DGC dataset. Some of these results were presented as abstracts at the 76th Scientific Sessions of the ADA in 2016 and the American Society of Human Genetics meeting in 2017.

Data availability DCCT data are available to authorised users at https:// repository.niddk.nih.gov and www.ncbi.nlm.nih.gov/projects/gap/cgi-bin/ study.cgi?study_id=phs000086.v3.p1. For Joslin 50-Year Medalist, CACTI, WESDR and EDC studies, the data are available on request from the authors.

Funding Industry contributors have had no role in the DCCT/EDIC study but have provided free or discounted supplies or equipment to support participants' adherence to the study: Abbott Diabetes Care (Alameda, CA, USA), Animas (Westchester, PA, USA), Bayer Diabetes Care (Tarrytown, NY, USA), Becton Dickinson (Franklin Lakes, NJ, USA), Eli Lilly (Indianapolis, IN, USA), Extend Nutrition (St Louis, MO, USA), Insulet Corporation (Bedford, MA, USA), LifeScan (Milpitas, CA, USA), Medtronic Diabetes (Minneapolis, MN, USA), Nipro Home Diagnostics (Ft Lauderdale, FL, USA), Nova Diabetes Care (Billerica, MA, USA), Omron (Shelton, CT, USA), Perrigo Diabetes Care (Allegan, MI, USA), Roche Diabetes Care (Indianapolis, IN, USA) and Sanofi (Bridgewater, NJ, USA). The DCCT/EDIC has been supported by cooperative agreement grants (1982-1993 and 2012-2017) and contracts (1982-2012) with the Division of Diabetes Endocrinology and Metabolic Diseases of the NIDKK (current grant nos. U01-DK-094176 and U01-DK-094157) and through support by the National Eye Institute, the National Institute of Neurologic Disorders and Stroke, the General Clinical Research Centers Program (1993-2007), and Clinical Translational Science Center Program (2006present), Bethesda, MD, USA.

The Joslin 50-Year Medalist study is supported by the NIDDK (P30DK036836, UL1 RR025758-03, R24 DK083957-01, DP3 DK094333-01, 1DP3DK112192-01), JDRF (17-2013-310) and 
contributions from Mr. T. Beatson (Beatson Foundation, Phoenix, AZ, USA) and the many participants.

Support for CACTI was provided by National Heart, Lung, and Blood Institute grants R01 HL113029, HL61753, HL79611, and HL113029, DERC Clinical Investigation Core P30 DK57516 and JDRF grant 172013-313. The study was performed at the Adult CTRC at University of Colorado Denver, CO, USA supported by NIH-M01-RR00051 and CTSA Grant UL1 TR001082, at the Barbara Davis Center for Childhood Diabetes and at the Colorado Heart Imaging Center (Denver, CO, USA). JKS-B was supported by an ADA Career Development Award (7-13-CD-50).

The WESDR was supported by grant R01EY016379 and NIH R01EY03083 from the National Eye Institute, NIH, and an unrestricted grant from Research to Prevent Blindness (New York, NY, USA).

The EDC study was supported by NIH grant R01-DK34818 and the Rossi Memorial Fund.

Funding for genotyping by Illumina $1 \mathrm{M}$ in DCCT was provided by NIH R01DK-077510 and genotyping by HumanCoreExome in all studies was provided by JDRF grant 17-2013-9.

DR was partly supported by a fellowship award from the Canadian Institutes of Health Research Strategic Training for Advanced Genetic Epidemiology (STAGE).

The study sponsor was not involved in the design of the study; the collection, analysis, and interpretation of data; writing the report; or the decision to submit the report for publication.

Duality of interest The authors declare that there is no duality of interest associated with this manuscript.

Contribution statement All named authors affirm that authorship is merited based on the International Committee of Medical Journal Editors (ICMJE) authorship criteria. DR designed the study, researched data and wrote the manuscript. RG-K, SBB, AJC, MGP, GLK, HAK, JKS-B, DMM, RK, BEKK, TJO, TC, MNW and RAO contributed to the data analysis and interpretation of the results, and reviewed/edited the manuscript. ADP designed the study, researched data, contributed to the discussion and reviewed/edited the manuscript. All authors approve the final version to be published. ADP is the guarantor of this work related to the DCCT/EDIC as well as the whole meta-GWAS; MGP, GLK and HAK are the guarantors of this work related to the Joslin 50-Year Medalist study; JKS-B and DMM are the guarantors of this work related to the CACTI study; RK and BEKK are the guarantors of this work related to the WESDR; and TJO and TC are the guarantors of this work related to the EDC study.

Open Access This article is distributed under the terms of the Creative Commons Attribution 4.0 International License (http:// creativecommons.org/licenses/by/4.0/), which permits unrestricted use, distribution, and reproduction in any medium, provided you give appropriate credit to the original author(s) and the source, provide a link to the Creative Commons license, and indicate if changes were made.

\section{References}

1. Gillespie KM, Gale EA, Bingley PJ (2002) High familial risk and genetic susceptibility in early onset childhood diabetes. Diabetes 51:210-214

2. Palmer JP, Fleming GA, Greenbaum CJ et al (2004) C-peptide is the appropriate outcome measure for type 1 diabetes clinical trials to preserve beta-cell function: report of an ADA workshop, 21-22 October 2001. Diabetes 53:250-264

3. Lachin JM, McGee P, Palmer JP (2014) Impact of C-peptide preservation on metabolic and clinical outcomes in the Diabetes Control and Complications Trial. Diabetes 63:739-748
4. Komulainen J, Kulmala P, Savola K et al (1999) Clinical, autoimmune, and genetic characteristics of very young children with type 1 diabetes. Childhood Diabetes in Finland (DiMe) Study Group. Diabetes Care 22:1950-1955

5. Wang L, Lovejoy NF, Faustman DL (2012) Persistence of prolonged C-peptide production in type 1 diabetes as measured with an ultrasensitive C-peptide assay. Diabetes Care 35:465-470

6. Faustman DL (2014) Why were we wrong for so long? The pancreas of type 1 diabetic patients commonly functions for decades. Diabetologia 57:1-3

7. Oram RA, Jones AG, Besser RE et al (2014) The majority of patients with long-duration type 1 diabetes are insulin microsecretors and have functioning beta cells. Diabetologia 57:187-191

8. McGee P, Steffes M, Nowicki M et al (2014) Insulin secretion measured by stimulated C-peptide in long-established type 1 diabetes in the Diabetes Control and Complications Trial (DCCT)/ Epidemiology of Diabetes Interventions and Complications (EDIC) cohort: a pilot study. Diabet Med 31:1264-1268

9. Steffes MW, Sibley S, Jackson M, Thomas W (2003) $\beta$-Cell function and the development of diabetes-related complications in the diabetes control and complications trial. Diabetes Care 26:832-836

10. Sorensen JS, Johannesen J, Pociot F et al (2013) Residual $\beta$-cell function 3-6 years after onset of type 1 diabetes reduces risk of severe hypoglycemia in children and adolescents. Diabetes Care 36:3454-3459

11. Kuhtreiber WM, Washer SL, Hsu E et al (2015) Low levels of Cpeptide have clinical significance for established type 1 diabetes. Diabet Med 32:1346-1353

12. Hoeldtke RD, Hampe CS, Bekris LM, Hobbs G, Bryner KD, Lernmark A (2007) Antibodies to GAD65 and peripheral nerve function in the DCCT. J Neuroimmunol 185:182-189

13. Keenan HA, Sun JK, Levine J et al (2010) Residual insulin production and pancreatic ss-cell turnover after 50 years of diabetes: Joslin Medalist Study. Diabetes 59:2846-2853

14. Onengut-Gumuscu S, Chen WM, Burren O et al (2015) Fine mapping of type 1 diabetes susceptibility loci and evidence for colocalization of causal variants with lymphoid gene enhancers. Nat Genet 47:381-386

15. Nakanishi K, Kobayashi T, Murase T et al (1993) Association of HLA-A24 with complete $\beta$-cell destruction in IDDM. Diabetes 42: 1086-1093

16. Lee JC, Biasci D, Roberts R et al (2017) Genome-wide association study identifies distinct genetic contributions to prognosis and susceptibility in Crohn's disease. Nat Genet 49:262-268

17. Oram RA, Patel K, Hill A et al (2016) A type 1 diabetes genetic risk score can aid discrimination between type 1 and type 2 diabetes in young adults. Diabetes Care 39:337-344

18. The DCCT Research Group (1986) The Diabetes Control and Complications Trial (DCCT). Design and methodologic considerations for the feasibility phase. Diabetes 35:530-545

19. The DCCT Research Group (1987) Effects of age, duration and treatment of insulin-dependent diabetes mellitus on residual $\beta$-cell function: observations during eligibility testing for the Diabetes Control and Complications Trial (DCCT). J Clin Endocrinol Metab 65:30-36

20. The Diabetes Control and Complications Trial Research Group (1998) Effect of intensive therapy on residual $\beta$-cell function in patients with type 1 diabetes in the diabetes control and complications trial. A randomized, controlled trial. Ann Intern Med 128:517-523

21. Dabelea D, Kinney G, Snell-Bergeon JK et al (2003) Effect of type 1 diabetes on the gender difference in coronary artery calcification: a role for insulin resistance? The Coronary Artery Calcification in Type 1 Diabetes (CACTI) Study. Diabetes 52:2833-2839

22. Garg SK, Moser EG, Bode BW et al (2013) Effect of sitagliptin on post-prandial glucagon and GLP-1 levels in patients with type 1 
diabetes: investigator-initiated, double-blind, randomized, placebocontrolled trial. Endocr Pract 19:19-28

23. Costacou T, Secrest AM, Ferrell RE, Orchard TJ (2014) Haptoglobin genotype and cerebrovascular disease incidence in type 1 diabetes. Diab Vasc Dis Res 11:335-342

24. He ZH, D'Eon SA, Tinsley LJ et al (2015) Cardiovascular disease protection in long-duration type 1 diabetes and sex differences. Diabetes Care 38:e73-e 74

25. Klein R, Klein BE, Moss SE, Cruickshanks KJ (1998) The Wisconsin Epidemiologic Study of Diabetic Retinopathy: XVII. The 14-year incidence and progression of diabetic retinopathy and associated risk factors in type 1 diabetes. Ophthalmology 105:1801-1815

26. Klein R, Knudtson MD, Lee KE, Gangnon R, Klein BE (2009) The Wisconsin Epidemiologic Study of Diabetic Retinopathy XXIII: the twenty-five-year incidence of macular edema in persons with type 1 diabetes. Ophthalmology 116:497-503

27. Klein R, Klein BE, Moss SE (1995) The Wisconsin Epidemiologic Study of Diabetic Retinopathy. XVI. The relationship of C-peptide to the incidence and progression of diabetic retinopathy. Diabetes 44:796-801

28. Paterson AD, Waggott D, Boright AP et al (2010) A genome-wide association study identifies a novel major locus for glycemic control in type 1 diabetes, as measured by both $\mathrm{A} 1 \mathrm{C}$ and glucose. Diabetes 59:539-549

29. Auton A, Brooks LD, Durbin RM et al (2015) A global reference for human genetic variation. Nature 526:68-74

30. McDonald JF, Moffitt RA (1980) The uses of Tobit analysis. Rev Econ Stat 62:318-32

31. R Core Team (2014) R: A language and environment for statistical computing. R Foundation for Statistical Computing, Vienna, Austria. URL http://www.R-project.org/. Accessed 1 Jul 2017

32. Willer CJ, Li Y, Abecasis GR (2010) METAL: fast and efficient meta-analysis of genomewide association scans. Bioinformatics 26 : 2190-2191

33. Pociot F, Akolkar B, Concannon P et al (2010) Genetics of type 1 diabetes: what's next? Diabetes 59:1561-1571
34. Noble JA, Valdes AM, Varney MD et al (2010) HLA class I and genetic susceptibility to type 1 diabetes: results from the type 1 diabetes genetics consortium. Diabetes 59:2972-2979

35. Winkler C, Krumsiek J, Buettner F et al (2014) Feature ranking of type 1 diabetes susceptibility genes improves prediction of type 1 diabetes. Diabetologia 57:2521-2529

36. Barker JM, Triolo TM, Aly TA et al (2008) Two single nucleotide polymorphisms identify the highest-risk diabetes HLA genotype: potential for rapid screening. Diabetes 57:3152-3155

37. Sun L, Craiu RV, Paterson AD, Bull SB (2006) Stratified false discovery control for large-scale hypothesis testing with application to genome-wide association studies. Genet Epidemiol 30:519-530

38. Yoo YJ, Bull SB, Paterson AD, Waggott D, Sun L (2010) Were genome-wide linkage studies a waste of time? Exploiting candidate regions within genome-wide association studies. Genet Epidemiol 34:107-118

39. Robertson CC, Onengut-Gumuscu S, Chen WM, Rich SS (2017) HLA imputation and allelic associations with type 1 diabetes in African Americans. In: American Society of Human Genetics Annual Meeting, Orlando, FL, USA

40. Jia X, Han B, Onengut-Gumuscu S et al (2013) Imputing amino acid polymorphisms in human leukocyte antigens. PLoS One 8:e64683

41. $\mathrm{Hu} \mathrm{X}$, Deutsch AJ (2015) Additive and interaction effects at three amino acid positions in HLA-DQ and HLA-DR molecules drive type 1 diabetes risk. Nat Genet 47:898-905

42. Nejentsev S, Howson JM, Walker NM et al (2007) Localization of type 1 diabetes susceptibility to the MHC class I genes HLA-B and HLA-A. Nature 450:887-892

43. Demeester S, Balke EM, Van der Auwera BJ et al (2016) HLA$A * 24$ carrier status and autoantibody surges posttransplantation associate with poor functional outcome in recipients of an islet allograft. Diabetes Care 39:1060-1064

44. Pruim RJ, Welch RP, Sanna S et al (2010) LocusZoom: regional visualization of genome-wide association scan results. Bioinformatics 26:2336-2337 